year available), there were 1281 large incidents ( $\geq 500$ affected individuals) and 164127 small incidents (<500 affected individuals). ${ }^{2-4}$ In aggregate, the large incidents affected approximately 52 million individuals while the small incidents affected 0.8 million individuals. These statistics suggest that, from a public policy perspective, large incidents have the potential to significantly impact more individuals than small incidents. Thus, larger breach incidents might deserve special attention from the government, industry, and research community. We do agree that all hospitals should take action to mitigate risk of data breaches and that smaller breaches also deserve more transparency because breached patient information does have human consequences, irrespective of the breach size where the information was compromised.

Drs Fabbri et al suggested that our results are affected by the HHS data collection process because only a PHI breach affecting 500 or more individuals requires HHS notification. We disagree. The HITECHA requires heath plan, health care providers, and business associates (ie, covered entities) to report all PHI breaches to HHS. There is no evidence, to our knowledge, that HHS has not been enforcing the law.

Drs Fabbri et al argued that hospitals that are unable to detect or did not actually detect data breach incidents are excluded from HHS public reporting. This is possible and consistent with the perception that the health care sector has not been an industry leader in information security. ${ }^{5}$ However, we believe that the number of these hospitals is likely to be limited because the vigilance of the public may compensate for these hospitals' lack of detection ability. Patients whose information is compromised usually complain to the hospital or HHS, facilitating the detecting and subsequent reporting of breaches. From 2003 to 2014, the HHS received 108031 complaints alleging covered entities disclosing patients' PHI inappropriately. ${ }^{6}$

Ge Bai, PhD, CPA

John (Xuefeng) Jiang, PhD

Renee Flasher, PhD, CPA

Author Affiliations: The Johns Hopkins Carey Business School, Washington, DC (Bai); Eli Broad College of Business, Michigan State University, East Lansing (Jiang); Miller College of Business, Ball State University, Muncie, Indiana (Flasher).

Corresponding Author: Ge Bai, PhD, CPA, The Johns Hopkins Carey Business School, Bernstein-Offit Bldg 353, 1717 Massachusetts Ave NW, Washington, DC 20036 (gbai@jhu.edu).

Conflict of Interest Disclosures: None reported.

1. Bai G, Jiang JX, Flasher R. Hospital risk of data breaches. JAMA Intern Med. 2017;177(6):878-880.

2. US Department of Health and Human Services. Annual Report to Congress on Breaches of Unsecured Protected Health Information For Calendar Years 2009 and 2010. https://www.hhs.gov/sites/default/files/ocr/privacy/hipaa /administrative/breachnotificationrule/breachrept.pdf. Accessed September 12, 2017.

3. US Department of Health and Human Services. Annual Report to Congress on Breaches of Unsecured Protected Health Information For Calendar Years 2011 and 2012. https://www.hhs.gov/sites/default/files/ocr/privacy/hipaa /administrative/breachnotificationrule/breachreport2011-2012.pdf. Accessed September 12, 2017.

4. US Department of Health and Human Services. Annual Report to Congress on Breaches of Unsecured Protected Health Information For Calendar Years
2013 and 2014. https://www.hhs.gov/sites/default/files/rtc-breach-20132014 .pdf. Accessed September 12, 2017.

5. Kwon J, Johnson ME. Proactive vs reactive security investments in the healthcare sector. Manage Inf Syst Q. 2014;38(2):451-471.

6. US Department of Health and Human Services. Annual Report to Congress on HIPAA Privacy, Security, and Breach Notification Rule Compliance For Calendar Years 2013 and 2014. https://www.hhs.gov/sites/default/files/rtc -compliance-20132014.pdf. Accessed September 12, 2017.

\section{Considerations for County-Level Inequalities in Life Expectancy}

To the Editor In an Original Investigation published in a recent issue of JAMA Internal Medicine, Dwyer-Lindgren and colleagues ${ }^{1}$ showed the existence and growth of alarmingly large inequalities in life expectancy across counties in the United States-up to a disturbing 20-year gap between the highest and lowest life expectancies between counties. I agree with the authors that these findings demand action because inequalities will continue to grow unabatedly in the face of inaction.

Dwyer-Lindgren and colleagues ${ }^{1}$ examined as mediators several demographic, socioeconomic, behavioral and metabolic, and health care factors, finding that they accounted for almost $75 \%$ of the variation in life expectancy. Importantly, the authors acknowledged the lack of other likely factors driving the inequalities observed. I believe diet quality, alcohol and drug abuse, and housing conditions are some of the critical missing factors in the analysis. ${ }^{2}$ In their Figure ${ }^{1}$ showing life expectancy by county, Baltimore City stands out in orange (life expectancy $\leq 75$ years) among its green and blue neighboring counties (life expectancy $\geq 80$ years). County-level data from the County Health Rankings project ${ }^{3}$ show that the proportion of the Baltimore City population that experiences food insecurity is $24 \%$, twice and thrice that observed in neighboring counties; $24 \%$ is also the proportion of households that experience dire housing problems like very high cost burden, severe overcrowding, and lack of kitchen or plumbing facilities-again much higher than in neighboring counties. Finally, with a residential segregation index of 69, Baltimore City remains the most racially segregated county in Maryland. ${ }^{3} \mathrm{Fu}-$ ture research must consider these critical behavioral and structural factors-particularly diet and housing conditions-as mediators of inequalities in life expectancy across counties.

Dwyer-Lindgren and colleagues ${ }^{1}$ also found that socioeconomic factors no longer explained the variation in life expectancy when all other factors were considered; thus, the authors suggested that the association between life expectancy and socioeconomic factors is largely mediated by behavioral and metabolic risk factors. I would like to caution against such conclusions, as evidence from large multicohort analyses ${ }^{4}$ indicates that the observed socioeconomic variation in allcause and cause-specific mortality-key factors in life expectancy-remains large and significant after behavioral and metabolic factors are accounted for. Doing so risks encouraging public health efforts and resources to focus on the individual, mistakenly ignoring the crucial role of structural forces that drive population and individual behavior, thereby hampering efforts to reduce inequalities in life expectancy.

\section{Carlos de Mestral, MSc}


Author Affiliation: Division of Chronic Diseases, Institute of Social and Preventive Medicine, Lausanne University Hospital, Lausanne, Switzerland.

Corresponding Author: Carlos de Mestral, MSc, Division of Chronic Diseases, Institute of Social and Preventive Medicine, Lausanne University Hospital, Route de la Corniche 10, 1010, Lausanne, Switzerland (carlos.de-mestral-vargas @chuv.ch).

Conflict of Interest Disclosures: None reported.

1. Dwyer-Lindgren L, Bertozzi-Villa A, Stubbs RW, et al. Inequalities in life expectancy among Us counties, 1980 to 2014: temporal trends and key drivers. JAMA Intern Med. 2017;177(7):1003-1011.

2. Marmot M, Friel S, Bell R, Houweling TAJ, Taylor S; Commission on Social Determinants of Health. Closing the gap in a generation: health equity through action on the social determinants of health. Lancet. 2008;372(9650):1661-1669.

3. County Health Rankings \& Roadmaps. 2017. http://www .countyhealthrankings.org. Accessed May 10, 2017.

4. Stringhini $S$, Carmeli C, Jokela M, et al; LIFEPATH consortium. Socioeconomic status and the $25 \times 25$ risk factors as determinants of premature mortality: a multicohort study and meta-analysis of 1.7 million men and women. Lancet. 2017:389(10075):1229-1237.

In Reply Our study ${ }^{1}$ on inequalities in life expectancy found that a combination of socioeconomic and race/ethnicity factors, behavioral and metabolic risk factors, and health care factors could explain $74 \%$ of the variation in life expectancy among counties. We agree with Dr Mestral that other factors such as diet quality and housing conditions may play a role in explaining the remaining variation. This is an important area of future research and will require identifying appropriate countylevel data sources that relate to these factors.

Our study ${ }^{1}$ also found that much of the effect at the county level of variation in socioeconomic factors on variation in life expectancy was mediated via risk factors. We do not interpret this to mean that risk factors should be the sole focus of efforts to improve population health. Socioeconomic factors are important, not only for health, but for well-being and prosperity more generally. Our research ${ }^{1}$ suggests that both socioeconomic and racial inequalities, as well as behavioral and metabolic risk factors, are important targets for improving population health and reducing inequalities. After all, individuals with low socioeconomic status are often the most impacted by these risk factors and are the most likely to benefit from such a strategy.

Ali H. Mokdad, PhD

Laura Dwyer-Lindgren, MPH

Christopher J. L. Murray, MD, DPhil

Author Affiliations: Institute for Health Metrics and Evaluation, University of Washington, Seattle.

Corresponding Author: Christopher J. L. Murray, MD, DPhil, Institute for Health Metrics and Evaluation, 2301 Fifth Ave, Ste 600, Seattle, WA 98121 (cjlm@uw.edu).

Conflict of Interest Disclosures: None reported.

1. Dwyer-Lindgren L, Bertozzi-Villa A, Stubbs RW, et al. Inequalities in life expectancy among US counties, 1980 to 2014: temporal trends and key drivers. JAMA Intern Med. 2017:177(7):1003-1011.

\section{Perplexing Conclusions Concerning Heat-Not-Burn Tobacco Cigarettes}

To the Editor While we welcome independent studies on our products, in a Research Letter published in a recent issue of JAMA Internal Medicine Auer et $\mathrm{al}^{1}$ described a chemical analysis of the IQOS aerosol that we find perplexing in several re- spects. Accuracy in science is, of course, always important. We believe that it is especially important in relation to potentially less harmful alternatives to cigarettes to ensure that adult smokers receive accurate information.

While some of the results reported by Auer et $\mathrm{al}^{1}$ seem consistent with those that we have previously published, significant points of difference in the described methodologies may account for the disagreements compared with our peerreviewed and published data. ${ }^{2}$ For example, the reported acrolein yield for the tested cigarette is 50 times below the level reported for similar cigarettes by Health Canada. ${ }^{3}$ It seems that Auer et $\mathrm{al}^{1}$ neither validated their methods with a standard reference cigarette nor compared their results with those published by a recognized regulatory agency. Further apparent methodological shortcomings are described in our full review of the article. ${ }^{4}$ Because we understand the importance of scientific rigor and the potential for skepticism around tobacco industry-generated data, we also commissioned an independent and accredited laboratory to analyze the IQOS aerosol. The relevant data were published on http://www.pmiscience.com and submitted as part of our Modified Risk Tobacco Product application to the US Food and Drug Administration. ${ }^{5}$

We have scientifically demonstrated the absence of combustion in IQOS. ${ }^{5,6}$ This has been corroborated by several combustion experts.

In their Research Letter, Auer et $\mathrm{al}^{1}$ did not present any data regarding the impact of IQOS use on indoor air quality. In contrast, we produced and published data using validated methods that show that the use of IQOS does not negatively impact indoor air quality according to international standards. ${ }^{6}$

We recognize the need to scrutinize the scientific work performed by the tobacco industry. It would seem equally important to scrutinize the work emanating from academia. We are therefore puzzled that the peer review system did not identify some of the methodological and interpretational findings outlined in our review. ${ }^{4}$

Philip Morris International transparently communicates its study results, ${ }^{6}$ including that IQOS yields over $90 \%$ lower levels of toxicants than the reference cigarette $3 \mathrm{R} 4 \mathrm{~F}$ and is not risk free. ${ }^{4-6}$ The totality of the evidence collected to date, across a broad range of toxicology, systems toxicology, and clinical studies, indicates that IQOS has the potential to present less risk of harm compared with continued smoking for adult smokers who switch to it completely. ${ }^{6}$

\section{Serge Maeder, PhD \\ Manuel C. Peitsch, PhD}

Author Affiliations: Philip Morris International Testing Laboratories \& Analytical Research, Switzerland (Maeder); Philip Morris International, Quai Jeanrenaud 5, 2000 Neuchâtel, Switzerland (Peitsch)

Corresponding Author: Manuel C. Peitsch, PhD, Philip Morris International, Quai Jeanrenaud 5, 2000 Neuchâtel, Switzerland (manuel.peitsch@pmi.com).

Conflict of Interest Disclosures: The authors are fully paid employees of Philip Morris International (PMI), the manufacturer of IQOS. All studies conducted by PMI with IQOS were solely funded by PMI.

1. Auer R, Concha-Lozano N, Jacot-Sadowski I, Cornuz J, Berthet A. Heat-not-burn tobacco cigarettes: smoke by any other name. JAMA Intern Med. 2017;177(7):1050-1052. doi:10.1001/jamainternmed.2017.1419 